\title{
Precisión y exhaustividad del registro de eventos adversos mediante una terminología de interfase
}

\author{
Accuracy and completeness of records of adverse events through interface terminology \\ Precisão e exaustividade no registro de eventos adversos usando uma terminologia de interface
}

Maribel González-Samartino ${ }^{1}$, Pilar Delgado-Hito ${ }^{1}$, Jordi Adamuz-Tomás ${ }^{2}$, Maria Fe Viso Cano², Mònica Castellà Creus ${ }^{3}$, María-Eulàlia Juvé-Udina ${ }^{1}$

Como citar este artículo:

González-Samartino M, Delgado-Hito P, Adamuz-Tomás J, Viso Cano MF, Castellà Creus M, Juvé-Udina ME. Accuracy and completeness of records of adverse events through interface terminology. Rev Esc Enferm USP. 2018;52:e03306. DOI: http://dx.doi.org/10.1590/S1980-220X2017011203306

${ }^{1}$ Universidad de Barcelona, Escuela de Enfermería, Barcelona, España.

${ }^{2}$ Hospital Universitario de Bellvitge, Barcelona, España.

${ }^{3}$ Instituto de Investigación Biomédica de Bellvitge, Barcelona, España.

\begin{abstract}
Objective: To determine what adverse events, including pressure ulcers, infection of the surgical site and aspiration pneumonia, nurses record in clinical histories, in terms of diagnostic accuracy and completeness, through ATIC. Method: Observational, descriptive, cross-sectional, multicenter study of 64 medical-surgical and semi-critical units of two university hospitals in Catalonia, Spain, during 2015. The diagnostic accuracy was assessed by means of the correspondence between the event declared in the Minimum Basic Data Set and the problem documented by the nurse. The record was considered complete when it contained the risk of the event, prescriptions of care and a record of the evolution. Results: The sample evaluated included 459 records. The accuracy results of pressure ulcers are highly correlated between the nursing diagnosis recorded and that declared in the Minimum Basic Data Set. The accuracy in surgical site infection is moderate, and aspiration resulting in pneumonia is very low. The completeness of results is remarkable, except for the risk of bronchoaspiration. Conclusion: The adverse event recorded by nurses with greatest accuracy is pressure ulcers.
\end{abstract}

\section{DESCRIPTORS}

Nursing Records; Pressure Ulcer; Surgical Wound Infection; Pneumonia Aspiration; Standardized Nursing Terminology; Patient Safety. 


\section{INTRODUCCIÓN}

Los pacientes que ingresan en los hospitales de agudos presentan un elevado nivel de complejidad que hace necesaria una prestación de cuidados altamente competente para prevenir complicaciones y minimizar eventos adversos $(\mathrm{EA})^{(1)}$.

Un EA es la consecuencia negativa de una atención que provoca lesiones no deseadas o una enfermedad que no ha sido evitada ${ }^{(2)}$ produciendo complicaciones de gravedad y comportando sufrimiento humano e incrementos de los costes financieros ${ }^{(3)}$. La incidencia de eventos adversos asociados a la hospitalización es del 9,2\%, siendo el 43.5\% considerados evitables ${ }^{(4)}$.

Entre las causas que pueden provocar un EA se han identificado factores humanos, ambientales, de equipamiento, del proceso de atención y de gestión de la información ${ }^{(5)}$. Según la naturaleza del problema se pueden clasificar en relación a los cuidados, la medicación, infecciones nosocomiales, procedimientos y/o diagnósticos ${ }^{(6)}$.

En las diferentes etapas del proceso de prestación de cuidados; una valoración inadecuada en el inicio de las complicaciones, una vigilancia insuficiente ${ }^{(7)}$, la omisión de cuidados $^{(8)}$ o el uso de lenguajes abstractos ${ }^{(9)}$ se han identificado como factores contribuyentes de EA.

Evidencias recientes indican que el uso de sistemas de lenguajes estandarizados como la clasificación de la North American Nursing Diagnosis Association (NANDA) ${ }^{(10)}$, tiene limitaciones significativas en la práctica a la hora de comunicar el cuidado de manera precisa. Se han descrito imprecisiones y ambigüedades en el uso de diagnósticos ${ }^{(9,11-12)}$, falta de conocimiento o comprensión de las etiquetas y un elevado nivel de abstracción de los conceptos que dificultan a la enfermera el poder reflejar la situación real del paciente o los cambios en su estado de salud ${ }^{(13)}$.

Los conceptos que representan el cuidado deben ser comprensibles y significativos para las enfermeras de manera que los diagnósticos, intervenciones y resultados sean reflejo del proceso de prestación de cuidados. De las diferentes opciones de lenguajes enfermeros estandarizados, ATIC es uno de los que presentan menores niveles de abstracción de sus conceptos. ATIC ${ }^{(14)}$ es "una terminología enfermera de interfase, multiaxial y orientada a conceptos, basada en el estudio del lenguaje natural que las enfermeras utilizan en su práctica diaria y revisada para su refinamiento teórico". Ha sido sometida a un proceso de evaluación de su validez cualitativa y de análisis de sus propiedades métricas ${ }^{(15-16)}$. ATIC se utiliza de forma habitual en los registros de historia clínica electrónica (HCE) de múltiples hospitales y centros sociosanitarios de la red pública de Catalunya (España) desde el año 2007 generando más de 200.000 registros de episodios de cuidados anuales. Ha sido reconocida como sistema de lenguaje que apoya el desarrollo y la práctica profesional enfermera en su rol autónomo y multidisciplinar por el Consejo General de Enfermería de España y en este momento está siendo evaluada para su reconocimiento por parte de otras organizaciones profesionales a nivel nacional e internacional.

Actualmente y pese la importancia de documentar los EA para garantizar la continuidad, calidad y seguridad de los cuidados, existe escasa investigación sobre qué informan las enfermeras en los registros electrónicos de salud a través de los lenguajes enfermeros estandarizados y el proceso de prestación de cuidados. La escasa bibliografía existente se centra en las úlceras por presión como único EA estudiado determinando, que la información documentada sobre el EA es poco precisa e incompleta ${ }^{(17-18)}$.

El objetivo principal de este estudio fue evaluar la precisión y la exhaustividad de la información sobre úlceras por presión (UPP), infección del sitio quirúrgico (ISQ) y aspiración con resultado de neumonía (AN) registrada por las enfermeras en la historia clínica electrónica mediante la terminología ATIC. Los objetivos secundarios fueron identificar si existen diferencias en el registro de los EA en función de formación específica y ámbito de prestación de cuidados.

\section{MÉTODO}

Esta investigación se planteó con un diseño observacional, descriptivo, transversal y multicéntrico. El ámbito de estudio incluyó 64 unidades médico-quirúrgicas de hospitalización y de cuidados semicríticos de dos hospitales universitarios de tercer nivel de Catalunya (España). Los datos se obtuvieron mediante la revisión de los registros electrónicos enfermeros entre enero y diciembre del 2015 de pacientes hospitalizados. Se incluyeron episodios de pacientes dados de alta, mayores de 18 años, en cuyo ingreso no habían requerido estancia en la unidad de cuidados intensivos y habían sufrido uno de los siguientes EA: úlcera por presión (UPP), infección de sitio quirúrgico (ISQ) y aspiración con resultado de neumonía (AN), declarados en el Conjunto Mínimo Básico de Datos (CMBD) de atención hospitalaria.

Se excluyeron episodios de pacientes de cirugías mayores ambulatorias y pacientes que presentaban el EA en el momento del ingreso.

La muestra se calculó con una frecuencia hipotética del $10 \%(\mathrm{p}=0.10)$ de eventos adversos, asumiendo un nivel de confianza del $95 \%(\alpha=0,05)$ y una precisión del $5 \%(\mathrm{i}=0.05)$, requiriéndose 138 episodios de cuidados para el análisis de cada EA. Se aplicó la fórmula de corrección para poblaciones finitas $n a=n /[1+(n / N)]$, determinándose que la muestra necesaria era de 153 episodios de cuidados por EA y una muestra final de 459 registros de episodios.

Se empleó una técnica de muestreo no probabilístico de tipo consecutivo.

Se evaluaron un total de 459 registros de episodios de cuidados de pacientes en los que se declaró a través del CMBD que habían presentado durante el periodo de hospitalización uno de los EA estudiados, correspondiendo n=153 episodios de cuidados de cada EA: a úlcera por presión, a infección de herida quirúrgica y aspiración con resultado se neumonía. La distribución de los registros de episodios, según los hospitales, fue la siguiente:

Hospital A. UPP ( $n=90)$, ISQ ( $n=91)$ y AN $(n=102)$

Hospital B. UPP $(n=63)$, ISQ $(n=62)$ y AN $(n=51)$.

Las variables principales del estudio fueron:

Precisión diagnóstica: correspondencia entre el registro del problema de salud documentado por la enfermera y el EA declarados en el CMBD (Cuadro 1). 
Cuadro 1 - Comunicación de los EA en CMBD y en ATIC en dos hospitales universitarios de Catalunya, España, 2015.

\begin{tabular}{|l|l|}
\hline $\begin{array}{l}\text { Evento Adverso comunicado en } \\
\text { CMBD }\end{array}$ & \multicolumn{1}{c|}{ Diagnostico ATIC } \\
\hline Úlcera por presión & $\begin{array}{l}\text { Úlcera por presión grado I } \\
\text { Úlcera por presión grado II } \\
\text { Úlcera por presión grado III } \\
\text { Úlcera por presión grado IV }\end{array}$ \\
\hline Infección sitio quirúrgico & $\begin{array}{l}\text { Herida quirúrgica } \\
\text { Herida quirúrgica contaminada }\end{array}$ \\
\hline $\begin{array}{l}\text { Aspiración con Neumonía } \\
\text { Aspiración con Neumonitis }\end{array}$ & $\begin{array}{l}\text { Broncoaspiración ** } \\
\text { Hipoxemia ** }\end{array}$ \\
\hline
\end{tabular}

* Con orientación de sospecha de infección registrada; presencia de signos y síntomas como calor, dolor, eritema, hinchazón y/o secreción purulenta.

* Con registro de la orientación de sospecha de Neumonía después de un episodio de aspiración; presencia de fiebre y/o secreciones purulentas.

\section{Definiciones operativas de los EA}

UPP: “Una úlcera por presión es una lesión localizada de la piel y/o tejido subyacente generalmente sobre una prominencia ósea, como resultado de presión en combinación con el cizallamiento"(19).

ISQ: "La infección superficial incisional que puede implicar la piel y el tejido subcutáneo, tejidos profundos como fascia y musculo u órganos y espacios que se manipularon durante la intervención” ${ }^{(20)}$.

AN: "Neumonía con factores previos de aspiración y aspiración demostrada o sospechada" (21).

Exhaustividad del registro: información pertinente y completa. Se consideró que el registro cumplía el criterio de exhaustividad cuando:

1. Se documentó el problema de riesgo en el plan de cuidados a partir de la valoración integral y focalizada mediante instrumentos sistematizados o escalas normalizadas.

2. Se registraron prescripciones de cuidados coherentes con el diagnóstico según las guías de práctica clínica y estándares internacionales ${ }^{(19-21)}$ en relación a los aspectos como:

UPP: prevención, soporte nutricional, reposicionamiento y movilización, dolor, cura, educación sanitaria, apoyo psicosocial.

ISQ: soporte nutricional, dolor, cura, educación sanitaria, apoyo psicosocial.

AN: control clínico, aspiración secreciones, ejercicios respiratorios, movilización precoz soporte nutricional, higiene oral y educación sanitaria.

3. Se registró la evolución de los cuidados en relación al EA a través de instrumentos estructurados o de las notas de progreso.

\section{LAS VARIABLES SECUNDARIAS FUERON:}

Formación metodológica: sesiones clínicas de cuidados consideradas reuniones entre miembros de un equipo enfermero en relación al análisis de un episodio de cuidados en un contexto clínico concreto y con un propósito analítico, evaluativo y reflexivo, a partir de la metodología y el vocabulario controlado ATIC. Los datos de esta variable se obtuvieron de los registros de episodios del hospital A, hospital donde se realizaban regularmente dicha sesiones.

Ámbito de prestación de cuidados: donde tiene lugar el EA: área médica o quirúrgica.

La investigación se realizó con la aprobación del proyecto por parte de los Comités Éticos de Investigación Clínica de los dos hospitales implicados (PRO34/15 CEIC 1495). Los datos personales tanto de pacientes como de profesionales se utilizaron según la normativa vigente en nuestro país, relativa a la confidencialidad de los datos. Dado el carácter observacional, descriptivo y retrospectivo del presente estudio, los Comités de Ética autorizaron la realización del estudio sin necesidad de consentimiento informado explícito.

Los datos se recogieron en un formulario Excel diseñado con las variables de estudio necesarias para la descripción de la muestra. Para el análisis al software estadístico SPSS (versión 18.0, SPSS Inc. Chicago, Illinois). La estrategia de análisis incluyó estadística descriptiva para identificar frecuencias, porcentajes, media y desviación típica. Se utilizó la prueba del Chi-cuadrado para las variables categóricas y el Test exacto de Fisher para las variables categóricas y cuantitativas. La significancia estadística se estableció con una $\mathrm{p}<0,05$ bilateral.

\section{RESULTADOS}

La media de edad de los pacientes con UPP fue de 72.8, con una estancia media de 38.7 días, la mayoría de los cuales (83\%) fueron hospitalizados en unidades médicas. De los episodios de los pacientes en los que se identificó la infección de herida quirúrgica como $\mathrm{EA}$, la hospitalización se produjo mayoritariamente en áreas quirúrgicas $121(79,1 \%)$, con una edad media de 64 años y una estancia media de 33,2 días. Los episodios de pacientes en los que se había declarado que habían presentado aspiración con resultado de neumonía, 117 pacientes estuvieron hospitalizados en áreas médicas $(76,5 \%)$, con una edad media de 72,9 años y una estancia media de 29,6 días. Otros datos como sexo, tipo de ingreso se muestran en la Tabla 1.

Tabla 1 - Características de la muestra de dos hospitales universitarios de Catalunya, España, 2015.

\begin{tabular}{llcccccc}
\hline & & \multicolumn{2}{c}{$\begin{array}{c}\text { UPP } \\
(\mathbf{n}=\mathbf{1 5 3})\end{array}$} & $\begin{array}{c}\text { ISQ } \\
(\mathbf{n}=\mathbf{1 5 3})\end{array}$ & \multicolumn{2}{c}{$\begin{array}{c}\text { AN } \\
(\mathbf{n}=\mathbf{1 5 3})\end{array}$} \\
\cline { 3 - 8 } & & $\mathbf{n}$ & $\%$ & $\mathbf{n}$ & $\%$ & $\mathbf{n}$ & $\%$ \\
\hline \multirow{2}{*}{ Sexo } & Hombres & 71 & 46,4 & 100 & 65,4 & 93 & 60,8 \\
& Mujeres & 82 & 53,6 & 53 & 34,6 & 60 & 39,2 \\
\hline \multirow{2}{*}{ Tipo de ingreso } & Urgente & 112 & 73,2 & 29 & 19 & 126 & 82,4 \\
& Programado & 41 & 26,8 & 124 & 81 & 27 & 17,6 \\
\hline \multirow{2}{*}{$\begin{array}{l}\text { Área de } \\
\text { hospitalización }\end{array}$} & Médica & 127 & 83 & 32 & 20,9 & 117 & 76,5 \\
& Quirúrgica & 26 & 17 & 121 & 79,1 & 36 & 23,5 \\
\hline Edad M (SD) & & 72,8 & $(15,3)$ & 64 & $(15,7)$ & 72,9 & $(14,3)$ \\
\hline Estancia M (SD) & & 38,7 & $(49)$ & 33,2 & $(21,7)$ & 29,6 & $(24,5)$ \\
\hline M - media; (SD) - desviación estándar. & & & & &
\end{tabular}




\section{PRECISIÓN DIAGNÓSTICA}

En los registros de los episodios de cuidados en los que se había declarado el EA de UPP ( $n=153)$, las enfermeras registraron un total de 157 diagnósticos en correspondencia con el EA. Los diagnósticos más prevalentes fueron el de Lesión por presión grado II $(39,2 \%)$ y Lesión por presión grado III $(35,3 \%)$.

En los episodios de cuidados correspondientes al EA de ISQ ( $\mathrm{n}=153$ ), las enfermeras registraron un total de 75 diagnósticos relacionados con el EA, 57 (37,2\%) registros del diagnóstico de Herida quirúrgica con especificaciones de infección y en 18 (11,7\%) el diagnóstico de Herida quirúrgica contaminada.

En relación a los registros acerca de la AN ( $\mathrm{n}=153)$, las enfermeras registraron el diagnóstico de hipoxemia en un $24,8 \%$ de los casos y el broncoaspiración en 2,6\%. (Tabla 2)

Tabla 2 - Características de la precisión diagnóstica en la comunicación de los EA en dos hospitales universitarios de Catalunya, España, 2015.

\begin{tabular}{llcc}
\hline EA & \multicolumn{1}{c}{ Diagnóstico ATIC } & $\mathbf{n}$ & $\%$ \\
\hline \multirow{2}{*}{ UPP $(\mathbf{n = 1 5 3 )}$} & Lesión por presión grado I & 25 & 16,6 \\
& Lesión por presión grado II & 60 & 39,2 \\
& Lesión por presión grado III & 54 & 35,3 \\
& Lesión por presión grado IV & 18 & 11,7 \\
\hline \multirow{2}{*}{ ISQ (n=153) } & Herida quirúrgica* & 57 & 37,2 \\
& Herida quirúrgica contaminada* & 18 & 11,7 \\
\hline \multirow{2}{*}{ AN (n=153) } & Hipoxemia** & 38 & 24,8 \\
& Broncoaspiración** & 4 & 2,6 \\
\hline
\end{tabular}

*Con orientación de sospecha de signos y síntomas de infección; calor, dolor, eritema, hinchazón y/o secreción purulenta.

** Con orientación de sospecha de Neumonía después de un episodio de aspiración; fiebre y/o secreciones purulentas.

\section{EXHAUSTIVIDAD}

En los registros de episodios de cuidados de los pacientes en los que se comunicó el EA de UPP se identificó el riesgo en 149 (97,4\%) episodios de cuidados. Las enfermeras registraron una media de 2,1 prescripciones de cuidados por diagnóstico relacionados con la UPP. Las prescripciones de cuidados más registradas fueron las referidas a la cura de la UPP estableciéndose Cura Compleja en $128(83,6 \%)$ de los episodios de cuidados y cura tópica en 59 (38,5\%). La evolución de los cuidados del EA se registró en 92 episodios de cuidados realizándose el mayor registro a través de las notas de progreso $57(37,2 \%)$.

En los episodios de cuidados de los pacientes en los que se determinó la Infección de herida quirúrgica, se identificó el riesgo en el plan de cuidados 150 (98\%) de los episodios de cuidados. La media de prescripciones de cuidados coherentes con el diagnóstico fue de 1,3 y las prescripciones más indicadas por las enfermeras tenían también relación con las curas. Siendo las prescripciones más numerosas la de cura tópica y cura compleja en el $63(41,2 \%)$ y 29 (19\%) de los episodios de cuidados. Las enfermeras registraron la evolución de los cuidados de este EA en 132 episodios de cuidados utilizando las notas de progreso para hacer el registro en la mayoría de los episodios de cuidados 80 (52,3\%).

En los registros de los episodios de cuidados pertenecientes al EA de neumonía por aspiración, las enfermeras identificaron el riesgo en el plan de cuidados solo 21 (13,7\%) de los episodios de cuidados. La media de prescripciones de cuidados por diagnóstico fue de 1,2 por episodio de cuidados. $Y$ las enfermeras prescribieron mayoritariamente cuidados relacionados con la administración de oxígeno en 42 (27,4\%) episodios de cuidados y en relación al control clínico del EA como control del patrón respiratorio en 36 (23,5\%) de los episodios. En este EA se ha registrado la evolución de los cuidados en 122 episodios de cuidados, registrándose en mayor número la evolución de los cuidados de forma estructurada $71(46,4 \%)$ de los episodios de cuidados (Tabla 3$)$.

Tabla 3 - Características de la exhaustividad en la comunicación de los EA en dos hospitales universitarios de Catalunya, España, 2015.

\begin{tabular}{|c|c|c|c|}
\hline EA & Características de exhaustividad & $\mathbf{n}$ & $\%$ \\
\hline \multirow{5}{*}{$\begin{array}{l}\text { UPP } \\
(n=153)\end{array}$} & Identificación del riesgo & 149 & 97,4 \\
\hline & $\begin{array}{l}\text { Prescripciones coherentes con el diagnóstico } \\
M(S D)\end{array}$ & 2,1 & $(1,9)$ \\
\hline & Evolución de los cuidados & & \\
\hline & En formulario estructurado & 35 & 22,8 \\
\hline & En notas de progreso & 57 & 37,2 \\
\hline \multirow{5}{*}{$\begin{array}{l}\text { ISQ } \\
(n=153)\end{array}$} & Identificación del riesgo & 150 & 98 \\
\hline & $\begin{array}{l}\text { Prescripciones coherentes con el diagnóstico } \\
M(S D)\end{array}$ & 1,3 & $(1,4)$ \\
\hline & Evolución de los cuidados & & \\
\hline & En formulario estructurado & 52 & 34 \\
\hline & En notas de progreso & 80 & 52,3 \\
\hline \multirow{5}{*}{$\begin{array}{l}\text { AN } \\
(n=153)\end{array}$} & Identificación del riesgo & 21 & 13,7 \\
\hline & $\begin{array}{l}\text { Prescripciones coherentes con el diagnóstico } \\
M(S D)\end{array}$ & 1,2 & $(1,8)$ \\
\hline & Evolución de los cuidados & & \\
\hline & En formulario estructurado & 71 & 46,4 \\
\hline & En notas de progreso & 51 & 33,3 \\
\hline
\end{tabular}

M - media; (SD) - desviación estándar.

\section{Diferencias de Registro del EA en relación A FORMACIÓN METODOLÓGICA}

Existen diferencias estadísticamente significativas en la comunicación de diagnósticos en la UPP $(74,2 \%$ registrados por las enfermeras que recibieron formación metodológica vs. $25,8 \%$ que no recibieron formación) con un $\mathrm{p}$ valor $<0.05$, en la ISQ $(67,8 \%$ vs. $22,2 \%)$ con un p valor $<0.05$ y la AN $(31,8 \%$ vs. $16,3 \%)$ con un $p$ valor $=0.03$. También se observan diferencias estadísticamente significativas en el registro de prescripciones de cuidados siendo en la UPP la media de prescripciones de 2,5 vs. 1,5 con un $\mathrm{p}$ valor $=0,01$, en la ISQ 
de 1,9 vs. 0,4 con un p valor $<0.05$ y en la AN de 1,4 vs. 0,7 con un $\mathrm{p}$ valor $=0,02$. En el registro de la evolución de los cuidados de los eventos adversos evaluados también existen diferencias estadísticamente significativas tanto en el registro de la evolución de forma estructurada como en el registro a través de las notas de progreso siendo el $\mathrm{p}$ valor $<0.05 \mathrm{en}$ todos los eventos adversos excepto en el registro de la evolución de los cuidados en la AN. En este evento aunque no existen diferencias estadísticamente significativas $(26,4 \%$ vs. $48,8 \%$, con un p valor $=0,07)$ se observa que las enfermeras que no recibieron formación específica registraron más la evolución de los cuidados a través de las notas de progreso que las enfermeras que recibieron formación metodológica.
Diferencias de Registro del EA en relación al ÁMBITO DE PRESTACIÓN DE CUIDADOS

No se observan diferencias estadísticamente significativas en el registro de los episodios de cuidados de ninguno de los eventos adversos evaluados en relación a si el paciente ha estado hospitalizado en un área médica o en un área quirúrgica excepto en el número de prescripciones de cuidados registrados por la enfermeras en el evento de $\mathrm{AN}$ en el que encontramos diferencias estadísticamente significativas registrando más prescripciones de cuidados las enfermeras del área quirúrgica que las enfermeras del área médica ( 1 vs. 1,8$) \mathrm{p}$ valor=0,03 (Tabla 4).

Tabla 4 - Diferencias en la comunicación del EA en relación a la asistencia a formación metodológica o al ámbito de prestación de cuidados en dos hospitales universitarios de Catalunya, España, 2015.

\begin{tabular}{|c|c|c|c|c|c|c|c|c|c|c|c|}
\hline \multirow{3}{*}{\multicolumn{2}{|c|}{ Comunicación del EA }} & \multicolumn{5}{|c|}{ Formación Especifica } & \multicolumn{5}{|c|}{ Ámbito de prestación de cuidados } \\
\hline & & \multicolumn{2}{|c|}{$\mathrm{Si}$} & \multicolumn{2}{|c|}{ No } & \multirow[b]{2}{*}{$\mathrm{pV}$} & \multicolumn{2}{|c|}{ U. Médica } & \multicolumn{2}{|c|}{ U. Quirúrgica } & \multirow[b]{2}{*}{ p V } \\
\hline & & $\mathbf{n}$ & $\%$ & $\mathbf{n}$ & $\%$ & & $\mathbf{n}$ & $\%$ & $\mathbf{n}$ & $\%$ & \\
\hline \multirow[t]{5}{*}{$\begin{array}{l}\text { UPP } \\
n=153\end{array}$} & Diagnóstico & 66 & 74,2 & 23 & 25,8 & 0,00 & 78 & 87,6 & 11 & 12,4 & 0,08 \\
\hline & Identificación del riesgo & 88 & 97,8 & 61 & 96,8 & 1,00 & 123 & 96,9 & 26 & 100 & 1 \\
\hline & Prescripciones M (SD) & 2,5 & $(1,9)$ & 1,5 & $(1,6)$ & 0,01 & 2,2 & $(1,9)$ & 1,6 & $(1,8)$ & 0,08 \\
\hline & Evolución estructurada & 37 & 58,7 & 15 & 16,7 & 0,00 & 42 & 33.1 & 10 & 38,9 & 0,37 \\
\hline & Evolución notas de progreso & 63 & 70 & 9 & 14,3 & 0,00 & 58 & 45,7 & 14 & 53,8 & 0,29 \\
\hline \multirow[t]{5}{*}{$\begin{array}{l}\text { ISQ } \\
n=153\end{array}$} & Diagnóstico & 61 & 67,8 & 14 & 22,2 & 0,00 & 18 & 24 & 57 & 76 & 0,42 \\
\hline & Identificación del riesgo & 88 & 97,8 & 62 & 94,8 & 1,00 & 38 & 100 & 112 & 97,4 & 0,57 \\
\hline & Prescripciones M(SD) & 1,9 & $(1,3)$ & 0,4 & $(0,9)$ & 0,00 & 1,6 & $(1,4)$ & 1,2 & $(1,3)$ & 0,120 \\
\hline & Evolución estructurada & 36 & 40 & 16 & 25,4 & 0,04 & 6 & 28,6 & 46 & 35,1 & 0,37 \\
\hline & Evolución en notas de progreso & 60 & 66,7 & 20 & 32,3 & 0,00 & 70 & 53,4 & 10 & 47,6 & 0,39 \\
\hline \multirow[t]{5}{*}{$\begin{array}{l}\text { AN } \\
n=153\end{array}$} & Diagnóstico & 35 & 31,8 & 7 & 16,3 & 0,03 & 30 & 25,6 & 12 & 33,3 & 0,24 \\
\hline & Identificación del riesgo & 33 & 30 & 4 & 9,3 & 0,05 & 28 & 23,9 & 9 & 25 & 0,52 \\
\hline & Prescripciones M(SD) & 1,4 & $(1,9)$ & 0,7 & $(1,5)$ & 0,02 & 1 & $(1,7)$ & 1,8 & $(2,1)$ & 0,03 \\
\hline & Evolución estructurada & 62 & 56,4 & 9 & 20,9 & 0,00 & 55 & 47 & 16 & 44,4 & 0,47 \\
\hline & Evolución en notas de progreso & 29 & 26,4 & 21 & 48,8 & 0,07 & 37 & 31,6 & 13 & 36,1 & 0,37 \\
\hline
\end{tabular}

M - media; (SD) - desviación estándar.

\section{DISCUSIÓN}

Los resultados de este estudio sugieren que la precisión diagnóstica de las enfermeras en cuanto a las UPP es elevada y la exhaustividad de los registros relacionados, notable. La precisión diagnóstica en la ISQ es moderada, a pesar de la exhaustividad del registro en relación al riesgo. En cambio, a pesar de que en la terminología ATIC existen conceptos diagnósticos es intervenciones específicas para la prevención y el abordaje de la NA, la precisión diagnóstica enfermera es baja, igual que la exhaustividad en relación a la identificación del riesgo.

\section{PreCISIÓN DIAGNÓSTICA}

Los resultados muestran una discrepancia entre el número de problemas de salud documentados por las enfermeras en los registros electrónicos y los declarados en el CMBD. Estas diferencias también se recogen en la literatura que evidencia que la comunicación de un evento adverso varía en función del profesional que lo comunica, su naturaleza y el grado de severidad ${ }^{(22)}$.

$\mathrm{La}$ integración de los lenguajes enfermeros en los registros electrónicos brinda a las enfermeras la oportunidad de mejorar la seguridad de los cuidados garantizando 
una comunicación eficaz pero para ello es necesario que los conceptos relativos a los cuidados se muestren representados de manera inequívoca. En la presente investigación se evidencia que frente a clasificaciones enfermeras internacionales como NANDA cuestionada por los elevados niveles de abstracción de sus $\operatorname{conceptos}^{(9,12)}$, la terminología ATIC ofrece a las enfermeras conceptos con diferentes niveles de especificidad que permiten reflejar de manera precisa problemas, situaciones, o respuestas reales o de riesgo ${ }^{(14)}$. En este estudio para comunicar los eventos de UPP y ISQ, las enfermeras han utilizado los diagnósticos ATIC de "Úlcera grado III", "Úlcera grado IV", "Herida quirúrgica", "Herida quirúrgica contaminada" que encuentran en NANDA un equivalente conceptual mucho más abstracto como es "Deterioro de la integridad tisular”. Los lenguajes enfermeros estandarizados tienen que asegurar que la información que se genera sea precisa y se pueda recuperar, compartir y reutilizar aportando comprensión y conocimiento al proceso de prestación de cuidados.

En relación al evento de UPP, los resultados de esta investigación son coincidentes con otros estudios que muestran que las enfermeras informan de un mayor número de UPP $(102,8 \%)$ que las que se comunican a través del $\mathrm{CMBD}^{(23)}$. Estos resultados difieren en la evaluación de la ISQy AN ya que a pesar de que ATIC ofrece conceptos diagnósticos con diferentes niveles de especificidad, su identificación como problema de salud en el registro electrónico es baja ISQ $(48,9 \%)$ y AN $(27,4 \%)$. La causas de este registro deficiente puede tener su origen en una falta de pericia en el reconocimiento del evento ${ }^{(24)}$ condi- $^{-}$ cionando la capacidad diagnóstica de la enfermera o por miedo a que el registro de un determinado juico clínico se pueda interpretar como una negligencia en el cuidado ${ }^{(25)}$.

\section{EXHAUSTIVIDAD}

La identificación del riesgo del problema de salud de los EA, presenta un registro desigual. En los episodios de cuidados de UPP e ISQ se identifica el riesgo en prácticamente la totalidad de los episodios de cuidados. Situación que podemos relacionar con la existencia de un proceso de ayuda a la toma de decisiones que no se produce en la AN dependiendo la identificación del riesgo exclusivamente, de la capacidad de razonamiento crítico de las enfermeras.

Todos los EA estudiados se comunican a través de la prescripción de cuidados presentando el registro más elevado de prescripciones el evento de UPP (Media=2,1). Dato que contrasta con una investigación sobre exhaustividad del registro de la UPP en la historia clínica electrónica que indicó que el $73 \%$ de las enfermeras no utilizó lenguaje estandarizado y documentó las intervenciones enfermeras en texto libre ${ }^{(26)}$. Según los autores las expresiones estandarizadas eran poco precisas y se alejaban del lenguaje utilizado habitualmente por las enfermeras. En el presente estudio las prescripciones de cuidados registradas para comunicar los eventos evaluados, UPP, ISQ y AN se muestran coherentes con los diagnósticos identificados y conformes con las guías de práctica clínica y estándares internacionales ${ }^{(19-21)}$, aunque se advierten insuficientes en relación a aspectos como soporte nutricional y educación sanitaria, careciendo también el evento de la AN de cuidados relacionados con la higiene oral y la movilización precoz.

La evolución de los cuidados no se registra de manera acorde con los problemas de salud identificados. Es decir, en los episodios de cuidados de UPP se registró la evolución de los cuidados únicamente en un $60 \%$ de los episodios en que se había diagnosticado el problema de salud mientras que en los otros eventos, se registró el seguimiento de los cuidados en un porcentaje mayor que el registro del problema de salud, en la ISQ $(86,3 \%)$ y en la AN $(79,7 \%)$. Por otro lado, cuando las enfermeras tienen que realizar descripciones específicas como por ejemplo características del tejido lesionado o del exudado, utilizan más el texto libre ${ }^{(26)}$. Esta situación en nuestra investigación no solo se circunscribe al evento de la UPP sino que también ocurre en la ISQ. Es importante trasladar a las enfermeras la importancia de registrar los cuidados siguiendo el proceso como columna vertebral de razonamiento clínico y como guía para mejorar la calidad y seguridad de los cuidados ${ }^{(18)}$.

Esta investigación aporta evidencia a favor de que las estrategias formativas mejoran la calidad de los registros ${ }^{(27)}$ ya que se constata que una intervención educativa específica en metodología aumenta significativamente la precisión diagnóstica y la exhaustividad en el registro de eventos adversos. Por el contrario no se observan diferencias significativas en la comunicación de los eventos en relación al área de hospitalización (médica o quirúrgica).

\section{CONCLUSIÓN}

Aunque los EA ligados a la hospitalización constituyen un importante problema de seguridad, existen escasas investigaciones que evalúen qué información registran las enfermeras a través del proceso de prestación de cuidados y los lenguajes enfermeros estandarizados.

Este estudio muestra una discrepancia entre el registro de los problemas de salud documentados por las enfermeras y los EA declarados en el CMBD. Las enfermeras registran con elevada precisión diagnostica el evento de UPP pero la precisión disminuye en el registro de la ISQ y AN.

La comunicación de los EA se evidencia exhaustiva en la identificación del riesgo del problema de salud cuando existen elementos de apoyo a las toma de decisiones y en las prescripciones de cuidados. Se constata que las prescripciones de cuidados se muestran exhaustivas. Es conveniente establecer estrategias que ayuden a mejorar el registro de la evolución de los cuidados a través de información estructurada que describa la prestación de cuidados.

La formación metodológica mejora la precisión diagnostica y la exhaustividad en la comunicación de los EA. Es sustancial complementar esta formación con acciones que refuercen la competencia profesional y el pensamiento crítico en la identificación de los EA. Y fomentar la comunicación siguiendo el proceso de prestación de cuidados como guía para mejorar la calidad y seguridad de los cuidados 


\section{RESUMEN}

Objetivo: Determinar qué registran las enfermeras en la historia clínica mediante ATIC, sobre los eventos adversos: úlceras por presión, infección del sitio quirúrgico y aspiración con resultado de neumonía, en términos de precisión diagnostica y exhaustividad. Método: Estudio observacional, descriptivo, transversal, y multicéntrico de 64 unidades médico-quirúrgicas y semicríticos de dos hospitales universitarios de Cataluña, España, durante el año 2015. La precisión diagnóstica se evaluó mediante la correspondencia entre el evento declarado en el Conjunto Mínimo Básico de Datos y el problema documentado por la enfermera. La exhaustividad se consideró cuando el registro contenía el riesgo del evento, prescripciones de cuidados y registro de la evolución. Resultados: La muestra evaluada fue de 459 registros. Los resultados de precisión de úlceras por presión muestran una elevada correspondencia entre el diagnóstico enfermero registrado y el declarado en el Conjunto Mínimo Básico de Datos. La precisión en la infección del sitio quirúrgico es moderada, y la aspiración con resultado de neumonía muy baja. Los resultados de exhaustividad son notables, excepto el riesgo de broncoaspiración. Conclusión: El evento adverso que registran las enfermeras con mayor precisión es la úlcera por presión.

\section{DESCRIPTORES}

Registros de Enfermería; Úlcera por Presión; Infección de la Herida Quirúrgica; Pneumonía por Aspiración; Terminología Normalizada de Enfermería; Seguridad del Paciente.

\section{RESUMO}

Objetivo: Determinar quais informações são registradas pelas enfermeiras nos registros eletrônicos por meio da terminologia ATIC, sobre os eventos adversos: úlceras de pressão, infecção de sítio cirúrgico e aspiração com resultado de pneumonia, em termos de precisão diagnóstica e de exaustividade. Método: Estudo observacional, descritivo, transversal e multicêntrico de 64 unidades médico cirúrgicas e semicríticas de dois hospitais universitários da Catalunha, Espanha, durante o ano 2015. A precisão diagnóstica foi avaliada pela correspondência entre o evento indicado no Conjunto Mínimo Básico de Dados e o registro de problemas documentados pela enfermeira. A exaustividade foi considerada quando o registro continha a identificação do risco do evento, as prescrições de cuidados e o registro da evolução. Resultados: A amostra avaliada foi de 459 registros. Os resultados em termos de precisão de úlceras de pressão mostram uma alta correlação entre o diagnóstico de enfermagem registrado e o diagnóstico declarado no Conjunto Mínimo Básico de Dados. A precisão na infecção de sítio cirúrgico é moderada, e a aspiração com resultado de pneumonia é muito baixa. Os resultados de exaustividade são notáveis, salvo o risco de broncoaspiração. Conclusão: O evento adverso que as enfermeiras registram com maior precisão é a úlcera de pressão.

\section{DESCRITORES}

Registros de Enfermagem; Lesão por Pressão; Infecção da Ferida Cirúrgica; Pneumonia Aspirativa; Terminologia Padronizada em Enfermagem; Segurança do Paciente.

\section{REFERENCIAS}

1. Despins LA. Organizational and individual attributes influencing patient risk detection. Clin Nurs Res. 2014;23(5):471-89. DOI:10.1177/1054773813490951

2. World Health Organization. Who draft guidelines for adverse event reporting and learning systems [Internet]. Geneva: WHO; 2008 [cited 2016 Sep 20]. Available from: http://apps.who.int/iris/bitstream/10665/69797/1/WHO-EIP-SPO-QPS-05.3-eng.pdf

3. Andersson $\AA$, Frank C, Willman AM, Sandman PO, Hansebo G. Adverse events in nursing: a retrospective study of reports of patient and relative experiences. Int Nurs Rev. 2015;62(3): 377-85. DOI: 10.1111/inr.12192

4. de Vries EN, Ramrattan MA, Smorenburg SM, Gouma DJ, Boermeester MA. The incidence and nature of in-hospital adverse events: a systematic review. Qual Saf Health Care. 2008;17(3):216-23.

5. Joint Commission. National Patients Safety Goals [Internet]. Illinois; 2016 [ cited 2016 Nov 15]. Available: https://www.jointcommission. org/topics/patient_safety.aspx

6. España. Ministerio de Sanidad y Consumo. Plan de Calidad para la Seguridad Nacional de Salud [Internet]. Madrid; 2006 [citado 2016 nov. 30]. Disponible en: http://www.seguridaddelpaciente.es/resources/contenidos/castellano/2006/ENEAS.pdf

7. Considine J, Mohr M, Lourenco R, Cooke R, Aitken M. Characteristics and outcomes of patients requiring unplanned transfer from subacute to acute care. Int J Nurs Pract. 2013;19(2):186-96. DOI: 10.1111/ijn.12056.

8. Maloney S, Fencl JL, Hardin SR. Nursing care missed? A comparative study of three North Carolina Hospitals. Medsurg Nurs. 2015;24(4):229-35.

9. Carrington JM. The usefulness of nursing languages to communicate a clinical event. Comput Inform Nurs. 2012;30(2):82-8.

10. Nanda International. Nursing diagnoses: definitions \& classification, 2015-2017. 10th ed. Oxford: Wiley Blackwell; 2014.

11. Morais SCRV, Nóbrega MML, Carvalho EC. Convergence, divergence and diagnostic accuracy in the light of two nursing terminologies. Rev Bras Enferm. 2015;68(6):777-83. DOl: http://dx.doi.org/10.1590/0034-7167.2015680613i

12. Giménez-Maroto AM, Serrano-Gallardo P. Imprecisiones del proceso diagnostico enfermero. Metas Enferm. 2009;11(10):57-62.

13. Park H, Lee E. Incorporating standardized nursing languages into an electronic nursing documentation system in korea: a pilot study. Int J Nurs Knowl. 2015;26(1):35-42. DOI: 10.1111/2047-3095.12038

14. Juvé-Udina ME. ATIC Eje diagnóstico. Barcelona: Naaxpot SLU; 2016.

15. Juvé-Udina ME. A nursing interface terminology: evaluation of face validity. OJN. 2012;2 (3):196-203. DOI: 10.4236/ojn.2012.23030

16. Juvé-Udina ME, Gonzalez-Samartino M, Matud-Calvo C. Mapping the diagnosis axis of an interface terminology to the NANDA International taxonomy. ISRN Nurs. 2012; 2012: 676905. DOI: 10.5402/2012/676905

17. Thoroddsen A, Sigurjónsdóttir G, Ehnfors M, Ehrenberg A. Accuracy, completeness and comprehensiveness of information on pressure ulcers recorded in the patient record. Scand J Caring Sci. 2013;27(1):84-91. DOI: 10.1111/j.1471-6712.2012.01004.x 
18. Cho I, Park H, Chung E. Exploring practice variation in preventive pressure-ulcer care using data from a clinical data repository. Intern J Med Inform. 2011;80(1):47-55. DOI: 10.1016/j.ijmedinf.2010.10.019

19. European Pressure Ulcer Advisory Panel; National Pressure Ulcer Advisory Panel; Pan Pacific Pressure Injury Alliance. Prevention and treatment of pressure ulcers: quick reference guide. Cambridge: Cambridge Media; 2014.

20. Healing Societies (WUWHS). Wound infection in clinical practice: an international consensus [Internet]. London: MEP; 2008 [cited 2016 Nov 30]. Available from: http://www.woundsinternational.com/media/issues/71/files/content_31.pdf

21. Torres O, Gil E, Pacho C, Ruiz D. Actualización de la neumonía en el anciano. Rev Españ Geriatr Gerontol. 2013;48(2):72-8. DOI:10.1016/j.regg.2012.06.001

22. Rafter N, Hickey A, Conroy RM, Condell S, O'Connor P, Vaughan D, et al. The Irish National Adverse Events Study (INAES): the frequency and nature of adverse events in Irish hospitals--a retrospective record review study. BMJ Qual Saf. 2017;26(2):111-9. DOI: $10.1136 /$ bmjqs-2015-004828

23. Sebastián-Viana T, González-Ruiz JM, Núñez-Crespo F, Lema-Lorenzo I, Gadea-Romero G, Losa-Iglesias ME. La validez de un registro clínico de úlceras por presión. An Sist Sanit Navar. 2014;37(1):17-24.

24. Purling A, King L. A literature review: graduate nurses' preparedness for recognising and responding to the deteriorating patient. J Clin Nurs. 2012;21(23-24):3451-65. DOI: 10.1111/j.1365-2702.2012.04348.x

25. Françolin L, Gabriel CS, Bernardes A, Silva AE, Brito MFP, Machado JP. Patient safety management from the perspective of nurses. Rev Esc Enferm USP. 2015;49(2):275-81. DOI: 10.1590/S0080-623420150000200013

26. Gunningberg L, Fogelberg-Dahm M, Ehrenberg A. Improved quality and comprehensiveness in nursing documentation of pressure ulcers after implementing an electronic health record in hospital care. J Clin Nurs. 2009;18(11):1557-64. DOI: 10.1111/j.1365-2702.2008.02647.x

27. Saranto K, Kinnunen UM, Kivekäs E, Lappalainen AM, Liljamo P, Rajalahti E, et al. Impacts of structuring nursing records: a systematic review. Scand J Caring Sci. 2014;28(4):629-47. DOI: 10.1111/scs.12094. 